\title{
MIXED WEAK TYPE INEQUALITIES FOR ONE-SIDED OPERATORS
}

\author{
FRANCISCO J. MARTÍN-REYES AND SHELDY J. OMBROSI
}

\begin{abstract}
We discuss mixed weak type inequalities in weighted spaces for one-sided operators. In particular, we prove that if $T_{c} f(x)=(x-$ $c)^{-1} \int_{c}^{x} f(y) d y, x>c$, is the Hardy averaging operator, $u \in A_{1}^{-}$(one-sided Muckenhoupt $A_{1}$ class) and $v \in A_{1}^{+}$(the another one-sided Muckenhoupt $A_{1}$ class) then there exists a constant $C$ such that $\sup _{c \in \mathbb{R}} \int_{\left\{x:\left|T_{c} f(x)\right|>v(x)\right\}} u v \leq$ $C \int_{\mathbb{R}}|f| u$.
\end{abstract}

\section{INTRODUCTION}

Let $T$ be a sublinear operator defined on measurable functions on $\mathbb{R}^{n}$, that is,

$$
|T(f+g)| \leq|T f|+|T g|, \quad|T(\lambda f)|=|\lambda||T f|,
$$

for all scalars $\lambda$ and all measurable functions $f$. A mixed weak type $(p, p)$ inequality for $T$ is an inequality of the form

$$
\int_{\{x:|T f(x)|>v(x)\}} u(x) v(x) d x \leq C \int|f(x)|^{p} w(x) d x,
$$

where $v, u$ and $w$ are nonnegative measurable functions and $C$ is independent of $f$. On one hand, this inequality contains the weighted weak type $(p, p)$ inequality, since if $v \equiv 1$ and we take the functions $f / \lambda, \lambda>0$, the above inequality becomes

$$
\int_{\{x:|T f(x)|>\lambda\}} u(x) d x \leq \frac{C}{\lambda^{p}} \int|f(x)|^{p} w(x) d x,
$$

that is, the weighted weak type $(p, p)$ inequality for the operator $T$ with respect to the weights $u$ and $w$. On the other hand, mixed weak type inequalities are related to the two weighted norm inequalities [2] and, probably, that is the reason why they are more difficult to handle than the corresponding weak type inequalities.

The research of the first author has been partially supported by Junta de Andalucía grant FQM 354 and spanish goverment grant MTM2005-08350-C03-02. The research of the second author has been partially supported by Universidad Nacional del Sur, grant SGCyT-UNS PGI 24/L058 and by a grant of spanish goverment MEC Res. 26/05/2006. 
Let $M$ be the Hardy-Littlewood maximal operator defined by

$$
M f(x)=\sup _{Q: x \in Q} \frac{1}{|Q|} \int_{Q}|f|
$$

where the supremum is taken over all cubes with sides parallel to the axis such that $x \in Q$. It is known that the weighted weak type $(1,1)$ inequality

$$
\int_{\{x: M f(x)>\lambda\}} u(x) d x \leq \frac{C}{\lambda} \int|f|(x) u(x) d x
$$

holds if and only if the weight $u$ satisfies the $A_{1}$ condition $\left(u \in A_{1}\right)$, that is, there exists $C>0$ such that

$$
M u(x) \leq C u(x) \quad \text { a.e. }
$$

Andersen and Muckenhoupt [2] proved the mixed weak type $(1,1)$ inequality

$$
\int_{\left\{x: M f(x)>|x|^{-d}\right\}}|x|^{-d} u(x) d x \leq C \int|f|(x) u(x) d x,
$$

under the assumptions $n=1, d \neq 1$ and $u \in A_{1}$. The same inequality was established for the Hilbert transform [2] and it was extended to singular integral operators in $\mathbb{R}^{n}[5]$. Sawyer [7] proved that the mixed inequality holds for some general non-power weights $v$. More precisely, he established that if $n=1$, $u \in A_{1}$ and $v \in A_{1}$ then

$$
\int_{\{x: M f(x)>v(x)\}} u(x) v(x) d x \leq C \int|f|(x) u(x) d x .
$$

The problem for the Hilbert transform was left open in that paper. Recently, the last inequality was proved [3] in $\mathbb{R}^{n}$ not only for $M$ but also and for singular integrals including the Hilbert transform.

This paper is devoted to the study of mixed weak type $(1,1)$ inequalities for one-sided operators. In the real line, the one-sided Hardy-Littlewood maximal operators $M^{-}$and $M^{+}$are defined by

$$
M^{-} f(x)=\sup _{h>0} \frac{1}{h} \int_{x-h}^{x}|f(x)| d x \quad \text { and } \quad M^{+} f(x)=\sup _{h>0} \frac{1}{h} \int_{x}^{x+h}|f(x)| d x .
$$

Weighted inequalities for $M^{-}$and $M^{+}$were studied first in [8] (see also [6]). It was established $[6]$ that the weighted weak type $(1,1)$ inequality

$$
\int_{\left\{x: M^{-} f(x)>\lambda\right\}} u(x) d x \leq \frac{C}{\lambda} \int|f|(x) u(x) d x
$$

holds if and only if the weight $u$ satisfies the $A_{1}^{-}$condition, that is, there exists $C>0$ such that

$$
M^{+} u(x) \leq C u(x) \quad \text { a.e. }
$$


The analogous result hold for $M^{+}$and $u \in A_{1}^{+}$which means $M^{-} u(x) \leq C u(x)$ almost everywhere. Arguing as in [7] we conjecture that the mixed weak type $(1,1)$ inequality

$$
\int_{\left\{x: M^{-} f(x)>v(x)\right\}} u(x) v(x) d x \leq C \int|f|(x) u(x) d x
$$

holds, under the assumptions $u \in A_{1}^{-}$and $v \in A_{1}^{+}$. In other words, the conjecture says that the mixed weak type $(1,1)$ inequality for $M^{-}$holds if $M^{-}$is of weak type $(1,1)$ with respect to $u(x) d x$ and $M^{+}$(the "adjoint" of $M^{-}$) is of weak type $(1,1)$ with respect to $v(x) d x$. So far, we have not been able to prove it. However we have found a proof of that inequality with $M^{-}$replaced by the Hardy averaging operators

$$
T_{c} f(x)= \begin{cases}\frac{1}{x-c} \int_{c}^{x} f(y) d y, & \text { if } x>c \\ 0, & \text { if } x \leq c .\end{cases}
$$

where $c$ is any fixed real number. Clearly, the operators $T_{c}$ are smaller than $M^{-}$and they are closely related to $M^{-}$since

$$
M^{-} f=\sup _{c \in \mathbb{R}} T_{c}|f| .
$$

For these operators we prove (see Corollary 2.8) that if $u \in A_{1}^{-}$and $v \in A_{1}^{+}$ then there exists a constant $C$ such that

$$
\sup _{c \in \mathbb{R}} \int_{\left\{x:\left|T_{c} f(x)\right|>v(x)\right\}} u v \leq C \int_{\mathbb{R}}|f| u
$$

for all measurable functions $f$. We obtain this result as a consequence of Theorem 2.6, where we state that the mixed weak type inequality holds for $T_{c}$ if $T_{c}$ is of weak type $(1,1)$ with respect to $u(x) d x$ and the formal adjoint $T_{c}^{*}$ is of weak type $(1,1)$ with respect to $v(x) d x$. In the next section we state and prove our results.

We shall use standard notations. In particular, if $E$ is a measurable set $E \subset \mathbb{R}$ then $|E|$ is the lebesgue measure of $E$.

\section{MiXed WEAK TYPE INEQUALITIES FOR HARDY OPERATORS}

We shall establish our results for the operators $T_{c}$ for any number $c$ but the proofs will be given in the case $c=0$, since the general case is proved in a completely similar way. In what follows, the Hardy operator $T_{0}$ will be denoted by $T$.

We start with a characterization of the mixed weak type inequality for $T_{c}$. The next theorem is essentially contained in [5] although in that paper a more general setting is considered and the Hardy operator is the one in $\mathbb{R}^{n}$ given by

$$
H f(x)=\frac{1}{|x|^{n}} \int_{B(0,|x|)} f(y) d y
$$


where $B(0,|x|)$ stands for the euclidian ball of center 0 and radius $|x|$. Observe that for $n=1$ the operator $H$ is the two-sided operator

$$
H f(x)=\frac{1}{|x|} \int_{-|x|}^{|x|} f(y) d y .
$$

We include the proof for completeness.

Theorem 2.1. Let $u$ and $v$ be nonnegative measurable functions defined on $\mathbb{R}$. Let $c \in \mathbb{R}$. The following statements are equivalent.

(a) There exists a constant $C$ such that

$$
\int_{\left\{x:\left|T_{c} f(x)\right|>v(x)\right\}} u v \leq C \int_{\mathbb{R}}|f| u
$$

for all measurable functions.

(b) There exists a constant $\widetilde{C}$ such that for all $a>c$

$$
\sup _{\lambda>0} \lambda \int_{\left\{x>a: \frac{1}{x-c}>\lambda v(x)\right\}} u v \leq \widetilde{C} u(x) \quad \text { for a.e. } x \in(c, a) .
$$

Further, if $C$ and $\widetilde{C}$ are the best constants in (a) and (b), respectively, then $\widetilde{C} \leq C \leq 4 \widetilde{C}$.

Proof. As we said above we work with $c=0$.

$(a) \Rightarrow(b)$. Let us fix $a>0$. Let $E$ be any measurable subset of $(0, a)$ and consider $f=\frac{1}{|E|} \chi_{E}$. If $x>a$ then

Therefore

$$
T f(x)=\frac{1}{x}
$$

$$
\int_{\left\{x>a: \frac{1}{x}>v(x)\right\}} u v \leq \int_{\{x: T f(x)>v(x)\}} u v \leq \frac{C}{|E|} \int_{E} u
$$

where the last inequality follows from statement (a). Since $E$ is any measurable subset of $(0, a)$, we obtain

$$
\int_{\left\{x>a: \frac{1}{x}>v(x)\right\}} u v \leq C \operatorname{ess} \inf \{u(x): x \in(0, a)\},
$$

which is (b) for $\lambda=1$. The inequality for all $\lambda$ follows in the same way since (a) holds for the pairs of functions $(u, \lambda v)$ for all $\lambda>0$ with the same constant.

$(b) \Rightarrow(a)$. We may assume without loss of generality that $f$ is integrable, $f \geq 0$ and $\int_{0}^{a} f>0$ for all $a>0$. Let $\left\{x_{n}\right\}_{n}$ be the decreasing sequence defined by $x_{0}=+\infty$ and

$$
\int_{0}^{x_{n+1}} f=\int_{x_{n+1}}^{x_{n}} f
$$


It is clear that $\lim _{n \rightarrow \infty} x_{n}=0$. If $x \in\left[x_{n+1}, x_{n}\right)$ then

$$
T f(x) \leq \frac{1}{x} \int_{0}^{x_{n}} f=\frac{4}{x} \int_{x_{n+2}}^{x_{n+1}} f .
$$

Therefore

$$
\{x: T f(x)>v(x)\} \subset \bigcup_{n=1}^{\infty}\left\{x \in\left[x_{n+1}, x_{n}\right): \frac{1}{x}>\frac{v(x)}{4 \int_{x_{n+2}}^{x_{n+1}} f}\right\} .
$$

If $\beta_{n}=\operatorname{ess} \inf \left\{u(x): x \in\left(0, x_{n+1}\right)\right\}$ we have by (b)

$$
\begin{aligned}
\int_{\{x: T f(x)>v(x)\}} u v & \leq 4 \widetilde{C} \sum_{n=1}^{\infty} \beta_{n} \int_{x_{n+2}}^{x_{n+1}} f \\
& \leq 4 \widetilde{C} \sum_{n=1}^{\infty} \int_{x_{n+2}}^{x_{n+1}} f u \leq 4 \widetilde{C} \int_{0}^{\infty} f u .
\end{aligned}
$$

Observe that taking $v=1$ in the theorem we obtain a characterization of the weights $u$ such that $T$ applies $L^{1}(u)$ into weak- $L^{1}(u)$. We state it as a corollary.

Corollary 2.2. Let $u$ be a nonnegative measurable functions defined on $\mathbb{R}$. Let $c \in \mathbb{R}$. The following statements are equivalent.

(a) There exists a constant $C$ such that

$$
\int_{\left\{x:\left|T_{c} f(x)\right|>\lambda\right\}} u \leq \frac{C}{\lambda} \int_{\mathbb{R}}|f| u
$$

for all measurable functions.

(b) $u$ satisfies $A_{1}\left(T_{c}\right)$, that is, there exists $\widetilde{C}>0$ such that for all $a>c$

$$
\sup _{y>a} \frac{1}{y-c} \int_{a}^{y} u \leq \widetilde{C} u(x) \quad \text { for a.e. } x \in(c, a) .
$$

Further, if $C$ and $\widetilde{C}$ are the best constants in (a) and (b), respectively, then $\widetilde{C} \leq C \leq 4 \widetilde{C}$.

The proof is direct from the theorem and the equality $\left\{x>a: \frac{1}{x-c}>\lambda\right\}=$ $\left(a, c+\frac{1}{\lambda}\right)$.

Remark 2.4. Notice that Andersen and Muckenhoupt [2] proved that statement (a) holds if and only if there exist $\alpha>0$ and $C(\alpha)$ such that for all $a>c$

$$
\int_{a}^{\infty}\left(\frac{a}{t-c}\right)^{\alpha} \frac{u(t)}{t-c} d t \leq C(\alpha) u(x) \text { for a.e. } x \in(c, a) .
$$

It is easy to see directly that this condition and $A_{1}\left(T_{c}\right)$ are equivalent. 
It can be proved also that the formal adjoint operator $T_{c}^{*}$ defined by

$$
T_{c}^{*} f(x)= \begin{cases}\int_{x}^{\infty} \frac{f(t)}{t-c} d t, & \text { if } x>c \\ 0, & \text { if } x \leq c,\end{cases}
$$

is of weak type $(1,1)$ with respect to the measure $v(x) d x$ if and only if $v \in$ $A_{1}\left(T_{c}^{*}\right)$, that is, there exists $C>0$ such that

$$
\frac{1}{x-c} \int_{c}^{x} v \leq C v(x) \text { for almost every } x>c .
$$

The proof is similar to the one for $T_{c}$ and we omit it (alternatively, the result can be obtained from the theorems in [2]). With the help of these conditions we can establish the mixed weak type inequality for $T_{c}$ for a wide class of weights.

Theorem 2.6. Let $u$ and $v$ be nonnegative measurable functions defined on $\mathbb{R}$. Let $c \in \mathbb{R}$. Assume that there exists $\varepsilon>0$ such that $u^{1+\varepsilon} \in A_{1}\left(T_{c}\right)$ and $v^{1+\varepsilon} \in A_{1}\left(T_{c}^{*}\right)$, i.e., there is a constant $C>0$ such that for all $a>c$

$$
\sup _{y>a} \frac{1}{y-c} \int_{a}^{y} u^{1+\varepsilon} \leq C u^{1+\varepsilon}(x) \quad \text { for a.e. } x \in(c, a) \text {, }
$$

and

$$
\frac{1}{x-c} \int_{c}^{x} v^{1+\varepsilon} \leq C v^{1+\varepsilon}(x) \quad \text { for almost every } x>c .
$$

Then there exists a constant $C$ such that

$$
\int_{\left\{x:\left|T_{c} f(x)\right|>v(x)\right\}} u v \leq C \int_{\mathbb{R}}|f| u
$$

for all measurable functions.

As a corollary we obtain our result for weights in the one-sided Muckenhoupt classes.

Corollary 2.8. Let $u$ and $v$ nonnegative measurable functions defined on $\mathbb{R}$. Assume that $u \in A_{1}^{-}$and $v \in A_{1}^{+}$. Then there exists a constant $C$ such that

$$
\sup _{c \in \mathbb{R}} \int_{\left\{x:\left|T_{c} f(x)\right|>v(x)\right\}} u v \leq C \int_{\mathbb{R}}|f| u
$$

for all measurable functions.

The corollary follows from the theorem, the easy implications $u \in A_{1}^{-} \Rightarrow u \in$ $A_{1}\left(T_{c}\right), v \in A_{1}^{+} \Rightarrow v \in A_{1}\left(T_{c}^{*}\right)$, and the well-known implications $u \in A_{1}^{-} \Rightarrow$ $u^{1+\varepsilon} \in A_{1}^{-}$and $v \in A_{1}^{+} \Rightarrow v^{1+\varepsilon} \in A_{1}^{+}$for some $\varepsilon>0$ (see $[8,6]$ ).

Proof of Theorem 2.6. We work with $c=0$. By Theorem 2.1, we only have to prove that

$$
\lambda \int_{\left\{x>a: \frac{1}{x}>\lambda v(x)\right\}} u v \leq C \operatorname{essinf}\{u(x): x \in(0, a)\}
$$


for all $a>0$ and all $\lambda>0$. Fix $\lambda>0$ and $a>0$ and set

$$
E=\left\{x>a: \frac{1}{x}>\lambda v(x)\right\}
$$

We may assume that $|E|>0$. Let us take any $z \in E$ such that

$$
\frac{1}{z} \int_{0}^{z} v^{1+\varepsilon} \leq C v^{1+\varepsilon}(z)
$$

We shall prove that

$$
\lambda \int_{E \cap(a, z)} u v \leq C \operatorname{essinf}(0, a) u
$$

Then letting $z$ tend to the essential supremum of $E$ we obtain the required inequality. Fix any number $\beta>1$ and choose $b \in(0, a)$ such that $b$ is a Lebesgue point of $u^{1+\varepsilon}$ and $u(b) \leq \beta\left(\operatorname{essinf}_{(0, a)} u\right)$. Now choose $\alpha$ such that $1-\varepsilon<\alpha<\frac{1}{1+\varepsilon}$. Applying the definition of $E$ and Hölder's inequality we obtain

$$
\begin{array}{r}
\int_{E \cap(a, z)} u v \leq \frac{1}{\lambda^{\alpha}} \int_{E \cap(a, z)} \frac{u(x)}{x^{\alpha}} v^{1-\alpha}(x) d x \\
\leq \frac{1}{\lambda^{\alpha}}\left(\int_{a}^{z} \frac{u^{1+\varepsilon}(x)}{x^{\alpha(1+\varepsilon)}} d x\right)^{\frac{1}{1+\varepsilon}}\left(\int_{a}^{z} v^{(1-\alpha) \frac{1+\varepsilon}{\varepsilon}}(x) d x\right)^{\frac{\varepsilon}{1+\varepsilon}} \\
\leq \frac{1}{\lambda^{\alpha}}\left(\int_{a}^{z} \frac{u^{1+\varepsilon}(x)}{x^{\alpha(1+\varepsilon)}} d x\right)^{\frac{1}{1+\varepsilon}}\left(\int_{a}^{z} v^{1+\varepsilon}(x) d x\right)^{\frac{1-\alpha}{1+\varepsilon}}(z-a)^{\frac{\varepsilon-1+\alpha}{1+\varepsilon}}
\end{array}
$$

Using (2.9), $z-a \leq z$ and $z \in E$ we obtain

$$
\begin{gathered}
\int_{E \cap(a, z)} u v \leq C \frac{z^{\frac{\varepsilon}{1+\varepsilon}}}{\lambda^{\alpha}}\left(\int_{a}^{z} \frac{u^{1+\varepsilon}(x)}{x^{\alpha(1+\varepsilon)}} d x\right)^{\frac{1}{1+\varepsilon}} v^{1-\alpha}(z) \\
\leq C \frac{z^{\alpha-\frac{1}{1+\varepsilon}}}{\lambda}\left(\int_{a}^{z} \frac{u^{1+\varepsilon}(x)}{x^{\alpha(1+\varepsilon)}} d x\right)^{\frac{1}{1+\varepsilon}}
\end{gathered}
$$

To estimate the last integral we take $c \in(b, a)$ and $f=\chi_{(b, c)}$. It is clear that for $x>a$

$$
T f(x)=\frac{c-b}{x} .
$$

Applying this equality

$$
\int_{a}^{z} \frac{u^{1+\varepsilon}(x)}{x^{\alpha(1+\varepsilon)}} d x=\frac{1}{(c-b)^{\alpha(1+\varepsilon)}} \int_{a}^{z}(T f(x))^{\alpha(1+\varepsilon)} u^{1+\varepsilon}(x) d x
$$


Since $u^{1+\varepsilon}$ satisfies $(2.3)$ we have that $T$ applies $L^{1}\left(u^{1+\varepsilon}\right)$ into weak- $L^{1}\left(u^{1+\varepsilon}\right)$. Therefore, by Kolmogorov's inequality (for instance, see [4])

$$
\begin{aligned}
& \int_{a}^{z} \frac{u^{1+\varepsilon}(x)}{x^{\alpha(1+\varepsilon)}} d x \\
& \quad \leq \frac{C}{(c-b)^{\alpha(1+\varepsilon)}}\left(\int_{a}^{z} u^{1+\varepsilon}(x) d x\right)^{1-\alpha(1+\varepsilon)}\left(\int_{b}^{c} u^{1+\varepsilon}(x) d x\right)^{\alpha(1+\varepsilon)} .
\end{aligned}
$$

Applying again the assumption on $u$ we have

$$
\int_{a}^{z} \frac{u^{1+\varepsilon}(x)}{x^{\alpha(1+\varepsilon)}} d x \leq C\left(\operatorname{essinf}_{(0, a)} u\right)^{(1+\varepsilon)(1-\alpha(1+\varepsilon))} z^{1-\alpha(1+\varepsilon)}\left(\frac{1}{c-b} \int_{b}^{c} u^{1+\varepsilon}\right)^{\alpha(1+\varepsilon)} .
$$

Since $c$ is any point in $(b, a)$ and $b$ is a Lebesgue point of $u^{1+\varepsilon}$, we get

$$
\left(\int_{a}^{z} \frac{u^{1+\varepsilon}(x)}{x^{\alpha(1+\varepsilon)}} d x\right)^{\frac{1}{1+\varepsilon}} \leq C\left(\operatorname{essinf}_{(0, a)} u\right)^{1-\alpha(1+\varepsilon)} z^{\frac{1}{1+\varepsilon}-\alpha} u^{\alpha(1+\varepsilon)}(b) .
$$

Now the property of $b$ gives

$$
\left(\int_{a}^{z} \frac{u^{1+\varepsilon}(x)}{x^{\alpha(1+\varepsilon)}} d x\right)^{\frac{1}{1+\varepsilon}} \leq C\left(\operatorname{essinf}_{(0, a)} u\right) z^{\frac{1}{1+\varepsilon}-\alpha} \beta^{\alpha(1+\varepsilon)} .
$$

Letting $\beta$ tend to 1 we obtain

$$
\left(\int_{a}^{z} \frac{u^{1+\varepsilon}(x)}{x^{\alpha(1+\varepsilon)}} d x\right)^{\frac{1}{1+\varepsilon}} \leq C\left(\operatorname{essinf}_{(0, a)} u\right) z^{\frac{1}{1+\varepsilon}-\alpha}
$$

This inequality together with (2.10) gives

$$
\int_{E \cap(a, z)} u v \leq \frac{C}{\lambda}\left(\operatorname{essinf}_{(0, a)} u\right)
$$

as we wished to prove.

Remark 2.13. We point out that $v \in A_{1}\left(T_{c}^{*}\right)$ does not imply $v^{1+\varepsilon} \in A_{1}\left(T_{c}^{*}\right)$ for some $\varepsilon>0$. We shall give an example because we have not found it in the literature.

Example 2.1. Let $I_{i}=\left(2^{i}+\frac{1}{2^{i}}, 2^{i}+1\right)$, for all natural number $i$, and $\Omega=$ $\cup_{i=1}^{\infty} I_{i}$. Now, we define

$$
w(x)=\chi_{\Omega^{c}}(x)+\sum_{i=1}^{\infty} \frac{\chi_{I_{i}}(x)}{\left(x-2^{i}\right)^{2}} d x .
$$

We shall see that $w \in A_{1}\left(T_{0}^{*}\right)$ and $w^{1+\varepsilon} \notin A_{1}\left(T_{0}^{*}\right)$ for any $\varepsilon>0$. Observe that $w \geq 1$. A simple computation gives

$$
\int_{I_{i}} w^{1+\varepsilon}=\int_{I_{i}} \frac{d x}{\left(x-2^{i}\right)^{2(1+\varepsilon)}}=\frac{1}{1+2 \varepsilon}\left(2^{i(1+2 \varepsilon)}-1\right) \sim 2^{i(1+2 \varepsilon)}
$$


We now show that $w$ satisfies $A_{1}\left(T_{0}^{*}\right)$. Let $x>2$ (since $w(y)=1$ for $y \leq 2$, for $x \leq 2$ it is easy), we choose a natural number $N$ such that $2^{N}<x \leq 2^{\bar{N}+1}$. It is enough to see that $\frac{1}{x} \int_{0}^{x} w$ is uniformly bounded, because $w(x) \geq 1$ for every $x$. We have that

$$
\frac{1}{x} \int_{0}^{x} w \leq \frac{1}{2^{N}} \int_{\Omega^{c} \cap\left(0,2^{N+1}\right)} w+\frac{1}{2^{N}} \int_{\Omega \cap\left(0,2^{N+1}\right)} w .
$$

Since $w(x)=1$ for $x \in \Omega^{c}$, the first summand is bounded by 2 and the second one is bounded by

$$
\frac{1}{2^{N}} \sum_{i=1}^{N} \int_{I_{i}} w \leq \frac{1}{2^{N}} \sum_{i=1}^{N} 2^{i} \leq 2 .
$$

Now, we will see that for any $\varepsilon>0, w^{1+\varepsilon}$ does not satisfy $A_{1}\left(T_{0}^{*}\right)$. Fix $\varepsilon>0$. If $x=2^{N}+s$ (with $\left.1 \leq s \leq 2\right)$ we have that $w(x)=1$, and by $(2.14)$ we have

$$
\frac{1}{x} \int_{0}^{x} w^{1+\varepsilon}>\frac{C}{2^{N}} \sum_{i=1}^{N} \int_{I_{i}} w^{1+\varepsilon} \geq \frac{C}{2^{N}} \sum_{i=1}^{N} 2^{i(1+2 \varepsilon)} \geq C 2^{2 N \varepsilon},
$$

which shows that $w^{1+\varepsilon}$ does not satisfy $A_{1}\left(T_{c}^{*}\right)$.

The same example shows that $u \in A_{1}\left(T_{c}\right)$ does not imply $u^{1+\varepsilon} \in A_{1}\left(T_{c}\right)$ for some $\varepsilon>0$. Keeping in mind this example, it is clear that the assumptions in Theorem 2.6 are stronger than $u \in A_{1}\left(T_{c}\right)$ and $v \in A_{1}\left(T_{c}^{*}\right)$. It is an open problem to know whether the conclusions of the theorem hold under these weaker assumptions. However, the answer is affirmative in the particular case of decreasing weights.

Theorem 2.15. Let $c \in \mathbb{R}$. Assume that $u$ is a decreasing weight in $(c, \infty)$ and the weight $v \in A_{1}\left(T_{c}^{*}\right)$. Then there exists a constant $C$ such that

$$
\int_{\left\{x:\left|T_{c} f(x)\right|>v(x) \mid\right\}} u v \leq C \int_{\mathbb{R}}|f| u
$$

for all measurable functions.

Proof. Assume $c=0$. As in the proof of Theorem 2.6, we only have to prove that

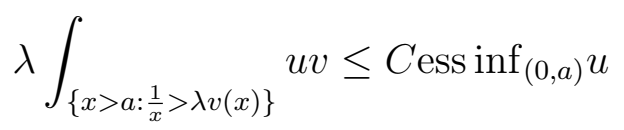

for all $a>0$ and all $\lambda>0$. Since $v \in A_{1}\left(T_{c}^{*}\right)$ we obtain that

$$
\left\{x>a: \frac{1}{x}>\lambda v(x)\right\} \subset\left\{x>a: \frac{C}{\lambda}>\int_{0}^{x} v\right\}=E_{\lambda} .
$$


Let $s_{0}=\sup \left\{x: x \in E_{\lambda}\right\}$. We have that $E_{\lambda} \subset\left(a, s_{0}\right)$ and $\int_{0}^{s_{0}} v \leq \frac{C}{\lambda}$. Using that $u$ is decreasing and we obtain

$$
\begin{aligned}
\lambda \int_{\left\{x>a: \frac{1}{x}>\lambda v(x)\right\}} u v & \leq \lambda\left(\operatorname{essinf}_{(0, a)} u\right) \int_{E_{\lambda}} v \\
& \leq \lambda\left(\operatorname{essinf}_{(0, a)} u\right) \int_{0}^{s_{0}} v \leq C \operatorname{essinf}_{(0, a)} u
\end{aligned}
$$

To finish the paper we show that for decreasing weights $u$, the natural condition $A_{1}^{+}$on the weight $v$ is sufficient to obtain the mixed weak type inequality for $M^{-}$.

Theorem 2.18. Let $u$ be decreasing in $\mathbb{R}$. Let $v \in A_{1}^{+}$. Then there exists $C>0$ such that

$$
\int_{\left\{x: M^{-} f(x)>v(x)\right\}} u v \leq C \int_{0}^{\infty}|f| u
$$

Proof. In fact, if $v \in A_{1}^{+}$then

$$
\left\{x: v(x)<M^{-} f(x)\right\} \subset\left\{x: M_{v}^{-}\left(f v^{-1}\right)(x)>\frac{1}{C}\right\},
$$

where

$$
M_{v}^{-} g(x)=\sup _{h>0} \frac{\int_{x-h}^{x}|g| v}{\int_{x-h}^{x} v}
$$

$\left(M_{v}^{+}\right.$is defined reversing the orientation in the real line). Now we recall $[1,6]$ that $M_{v}^{-}$applies $L^{1}(u v)$ into weak- $L^{1}(u v)$ if and only if $M_{v}^{+} u \leq C u$ almost everywhere. It is clear that $\mathrm{u}$ satisfies that condition because $u$ decreases. Therefore,

$$
\int_{\left\{x: M^{-} f(x)>v(x)\right\}} u v \leq \int_{\left\{x: M_{v}^{-}\left(f v^{-1}\right)(x)>\frac{1}{C}\right\}} u v \leq C \int_{\mathbb{R}}|f| v^{-1} u v=C \int_{\mathbb{R}}|f| u,
$$

as we wanted to prove.

\section{REFERENCES}

[1] K. Andersen, Weighted inequalities for maximal functions associated with general measures, Trans. Amer. Math. Soc. 326 (1991), no. 2, 907-920.

[2] K.F. Andersen, B. Muckenhoupt, Weighted weak type Hardy inequalities with applications to Hilbert transforms and maximal functions, Studia Math. 72 (1982), no. 1, 9-26.

[3] D. Cruz-Uribe, J.M. Martell and C. Pérez, Weighted weak-type inequalities and a conjecture of Sawyer, Int. Math. Res. Not. 2005, no. 30, 1849-1871.

[4] J. García-Cuerva, J.L. Rubio de Francia Weighted Norm Inequalities and Related Topics, Mathematics Studies. (1985).

[5] F.J. Martín-Reyes, P. Ortega Salvador and M.D. Sarrión Gavilán, Boundedness of operators of Hardy type in $\Lambda^{p, q}$ spaces and weighted mixed inequalities for singular integral operators, Proc. Roy. Soc. Edinburgh Sect. A 127 (1997), no. 1, 157-170. 
[6] F.J. Martín-Reyes, P. Ortega Salvador and A. de la Torre, Weighted inequalities for onesided maximal functions, Trans. Amer. Math. Soc. 319 (1990), no. 2, 517-534.

[7] E. Sawyer, A weighted weak type inequality for the maximal function. Proc. Amer. Math. Soc. 93 (1985), no. 4, 610-614.

[8] E. Sawyer, Weighted inequalities for the one-sided Hardy-Littlewood maximal functions. Trans. Amer. Math. Soc. 297 (1986), no. 1, 53-61.

(Francisco J. Martín-Reyes) Departamento de Análisis Matemático, Facultad de Ciencias, Universidad de Málaga, 29071 Málaga, Spain

E-mail address: martin_reyes@uma.es

(Sheldy J. Ombrosi) Departamento de Matemática, Universidad Nacional del Sur, Bahía Blanca, 8000, Argentina

Current address: Departamento de Análisis Matemático, Facultad de Matemáticas, Universidad de Sevilla, 41080 Sevilla, Spain

E-mail address: sombrosi@uns.edu.ar 